\title{
Issues and Challenges in Sedentary Behavior Measurement
}

\author{
Minsoo Kang \\ Department of Health and Human Performance, Middle Tennessee State University, Murfreesboro,
} 5 Tennessee

David A. Rowe

School of Psychological Sciences and Health, University of Strathclyde, Glasgow, UK 
Issues and Challenges 2

\begin{abstract}
Previous research has shown the negative impact of sedentary behavior on health, including cardiovascular risk factors, chronic disease-related morbidity, and mortality. Accurate measurement of sedentary behavior is thus important to plan effective interventions and to inform public health messages. This paper a) provides an overview of the nature and importance of sedentary behavior, b) describes measurement methods, including subjective and objective measurement tools, c) reviews the most important measurement and data processing issues and challenges facing sedentary behavior researchers, and d) presents key findings from the most recent sedentary behavior measurement-related research. Both subjective and objective measures of sedentary behavior have limitations for obtaining accurate sedentary behavior measurements compliant with the current definitions of sedentary behavior, especially when investigating sedentary behavior as part of the full spectrum of physical behaviors. Regardless of the sedentary behavior measure chosen, researchers must be aware of all possible sources of error inherent to each technique and minimize those errors thereby increasing validity of the outcome data.
\end{abstract}

Keywords: validity, objective measure, subjective measure, accelerometer, public health, physical activity 
The chronology of population health recommendations associated with human movement could be generally characterized as (a) do planned, structured exercise in order to improve fitness (American College of Sports Medicine, 1978); (b) incorporate lifestyle activities of moderate to vigorous intensity on 30 minutes per day (MVPA; Pate et al., 1995); and (c) accumulate lifestyle activities of moderate to vigorous intensity for 150 minutes per week (US Physical Activity Guidelines Committee, 2008). More recently, population public health recommendations have emerged for sedentary behavior in Canada (Canadian Society for Exercise Physiology, 2012; Tremblay et al., 2011) and Australia (Australian Government Department of Health, 2014; Brown, Bauman, Bull, \& Burton, 2012).

Sedentary behavior is a major health risk, independent of being physically inactive (i.e., obtaining an insufficient amount of MVPA). For example, sedentary behavior has been demonstrated to be an independent risk factor for poor health and premature death (Tremblay, Colley, Saunders, Healy, \& Owen, 2010). Even for those who meet the public health recommendations for physical activity, too much sitting can compromise metabolic health (Healy, Dunstan, Salmon, Shaw, et al., 2008; Salmon et al., 2011). Thus, the empirical evidence supports the conceptual portrayal of sedentary behavior (too much sitting with low energy expenditure) as a separate and independent construct from inactivity (doing insufficient health-enhancing physical activity). Considering that sitting is the most common form of sedentary behavior and that people spend about 55\% of their time in sedentary behavior during the day (Matthews et al., 2008), reducing sitting time has become an important public health strategy for chronic disease prevention.

Accurate measurement of sedentary behavior is critical to determining the relationship between sedentary behavior and health, to planning effective interventions, and to informing public health messages. As population life expectancy increases, it is crucial to extend current 
knowledge on the appropriate measurement of sedentary behavior in health outcome research (Rosenberger, 2012). Continued efforts are being made to reduce measurement error in assessing sedentary behavior, but due to the changing definitions of sedentary behavior and the challenges of capturing its two primary components (posture and energy expenditure), many critical measurement challenges remain unresolved.

The purpose of this article is to provide an overview of the nature and importance of sedentary behavior, to describe the primary measurement methods including subjective and objective measurement tools, to review important sedentary behavior measurement and data processing issues and challenges, and to present key findings from the most recent sedentary behavior measurement-related research. It concludes with a list of recommendations to be considered when measuring sedentary behavior.

What is sedentary behavior?

The definition of sedentary behavior has evolved considerably in the past 10 years. Interestingly, its roots lie in the Latin sedere, meaning "to sit". In early physical activity recommendations and physical activity epidemiology research, the term "sedentary" (or sometimes "essentially sedentary") was synonymous with "inactive/low active" (e.g., Paffenbarger, Hyde, Wing, \& Hsieh, 1986), or "inactive/irregularly active" (e.g., Centers for Disease Control and Prevention, 1993). Predominant current thinking is that inactivity and sedentariness are separate constructs. Thus, Owen, Healy, Matthews, and Dunstan (2010) proposed sedentariness to be defined that "sedentariness (too much sitting) is distinct from too little exercise" (p. 105). More specifically, this newer conceptualization has been characterized as prolonged sitting, requiring low levels of energy expenditure ranging from 1.0 to 1.5 metabolic equivalent units (METs) (Owen, Bauman, \& Brown, 2009; Pate, O'Neill, \& Lobelo, 2008). In 2012, the Sedentary Behavior Research Network published an updated 
definition of sedentary behavior as "any waking behavior characterized by energy expenditure $\leq 1.5$ METs while in a sitting or reclining posture" (p. 540). From these sources, there appears to be a broad contemporary consensus that sedentariness involves both a postural aspect (sitting or lying) and low levels of energy expenditure, and does not include light activity (e.g., quiet standing).

Energy expenditure or the amount of movement is relatively homogenous during sedentary behavior whereas physical activity has several intensity categories and movement patterns (such as upper body, whole-body, ambulatory, and stationary). In some aspects the physical mode of sedentary behavior also is far more homogenous (sitting or lying), compared to physical activity. Perhaps the more challenging aspect of measuring sedentary behavior is that its temporal pattern is far more complex, because it occurs throughout the day and is broken up into multiple bouts of varying length making the temporal patterning aspect of sedentary behavior the most difficult to interpret.

From a pure movement or energy expenditure perspective, Tremblay et al. (2010) conceptualized sedentary behavior as being at one end of a physiological continuum, above sleep, with vigorous intensity physical activity at the opposite end of the continuum. Tremblay et al. (2010) also described the components of sedentary behavior using the acronym SITT (Sedentary bout frequency, Interruptions of sedentary behavior, Time spent in sedentary behavior, and Type of behavior engaged in while being sedentary). Although not directly analogous to the FITT (Frequency, Intensity, Time, and Type) dimensions associated with health-enhancing physical activity, it serves as a useful heuristic and reminder throughout this paper of the importance of matching conceptualization of the construct to the methods used to measure it.

Overview of Sedentary Behavior Measurement Methods 
Sedentary behavior has been evaluated using a variety of subjective measures (questionnaire, interview, and activity-recall instruments) and objective measures (accelerometry-based motion sensors and inclinometers). Several current approaches to measuring sedentary behavior match dimensions represented by the SITT acronym (Tremblay et al., 2010). Sedentary bout frequency (S) can be derived from any objective measure of sedentary behavior and sedentary breaks. Similarly, interruptions (I) can be operationalized either through sit-to-stand transitions from instruments such as the activPAL, or by detecting any drop in activity counts below a specific threshold (e.g., 100 counts/minute $[\mathrm{cpm}]$ in the ActiGraph). Total time spent in sedentary behavior ( $\mathrm{T}$ ) can be derived by summing time in sedentary bouts, via procedures just described, or via questionnaires. For example, the Occupational Sitting and Physical Activity Questionnaire (Chau et al., 2012) uses an algorithm to multiply self-reported percent occupational time spent sitting by self-reported total time spent at work. Lastly, type of behavior engaged in while being sedentary $(\mathrm{T})$ cannot typically be derived from objective measures of sedentary behavior, but is conveniently assessed via self-report. More recently, wearable cameras and photographic images have been used to classify contextual information about specific behaviors while sitting (e.g., Chastin, Schwartz, \& Skelton, 2013; Kerr et al., 2013; Kim, Barry, \& Kang, 2015), providing a relatively nonintrusive method of directly observing behavior type and context.

\section{Subjective Measures of Sedentary Behavior}

A common approach to evaluating sedentary behavior is to ask a single question (e.g., time spent TV viewing) in an interview or activity-based questionnaire format concerning the amount of total time spent sitting or lying down (e.g., Clemes, David, Zhao, Han, \& Brown, 2012). Because this approach provides only general information regarding the sedentary lifestyle of an individual, it may not be a complete representation of sedentary behavior and 
thus, it can be challenging for researchers or health professionals to develop targeted behavior change intervention programs to reduce sedentary behaviors based on this type of evidence. Put simply, basic summary measures provide insufficient data to inform strategies for intervening in a complex set of behaviors.

Researchers have recently developed measurement tools that assess multiple sedentary behaviors (e.g., TV viewing, reading, screen time) and domain-specific sedentary behaviors (e.g., sitting at work or at home, transportation) (Chau, van der Ploeg, Dunn, Kurko, \& Bauman, 2012; Marshall, Miller, Burton, \& Brown, 2010; Rosenberg et al., 2010). The Sedentary Behavior Questionnaire (Rosenberg et al., 2010) and Marshall Sitting Questionnaire (Marshall et al., 2010) assess time spent being sedentary on weekdays and weekend days. The Sedentary Behavior Questionnaire is designed to assess the amount of time spent in nine behaviors (e.g., watching TV, sitting and talking on the phone, driving/riding in a car, bus or train), and the Marshall Sitting Questionnaire consists of reports of time spent sitting in five domains (e.g., while at work, while using a computer at home). Because the tools describe patterns of sedentary behavior throughout an entire week including the weekend, individualized and targeted interventions can more effectively target time spent in sedentary pursuits. The Occupational Sitting and Physical Activity Questionnaire (Chau et al., 2012) is specific to the workplace environment and asks participants to report how many hours they worked in the previous 7 days and the number of days they were at work. It subsequently asks for percent time at work spent sitting, standing and in physical activity. A basic algorithm is then used to compute total sitting time at work during one week.

Subjective measures have historically been preferred in large-sample observational studies of physical activity due to relatively low administration costs and participant burden 
(Sallis \& Saelens, 2000), and the same has recently been true for use of surveys for largesample measurement of sedentary behavior (Atkin et al., 2012). Subjective measures are also useful to identify the type of behavior and the context in which the sedentary behavior occurs, which is typically not possible with objective measurement methods. The reported validity coefficients of subjective measures, however, have varied greatly among studies (range -.02 to .83; Healy, Clark, et al., 2011). Depending on the study, estimates from subjective measures may over- or underestimate time spent in sedentary behavior (Clark et al., 2011; Healy, Clark, et al., 2011). Many factors may lead to the inconsistency, including inappropriate criterion measures used (e.g., using motion sensors instead of using direct observation), different qualitative attributes (e.g., recall period and question/response format), mode of administration (e.g., interview vs. self-administration), the time frame of assessment (e.g., past day, past week, usual week, past year), population being assessed (e.g., children, adults and older adults), and cultural norm and social desirability of the response (Atkin et al., 2012; Healy, Clark, et al., 2011). Furthermore, sedentary behavior is not commonly structured and purposive like physical activity; rather, it occurs persistently throughout the day. This may negatively impact participants' ability to recall accurately the amount of time spent in sedentary behaviors in free-living environments (Healy, Clark, et al., 2011; Owen et al., 2010).

To overcome some of these recall-related problems, diaries and ecological momentary assessment (EMA) were developed to record patterns (i.e., the temporal combination of activities) of sedentary behaviors. EMA is a strategy that can simultaneously capture a behavior and the factors that may influence it by allowing participants to report their current activity, location, and social surroundings (Dunton, Liao, Intille, Spruijt-Metz, \& Pentz, 2011). One of the major advantages is its ability to provide ecologically valid ("real-world") 
information on an individual's sedentary behavior patterns. Other advantages include the small expense in data collection and the ability to administer to many participants at the same time.

Limitations of the diary and EMA method are the relatively heavy participant burden, since they both require a high level of adherence to reporting protocols, and the potential for participant reactivity (Atkin et al., 2012). Reactivity (sometimes referred to as the Hawthorne Effect; Campbell \& Stanley, 1966) is the phenomenon of altering natural behavior patterns purely as a result of measuring the behavior. Thus, regular self-monitoring may increase a participant's awareness of their sedentary behavior, which in turn may cause them to alter their behavior by, for example, breaking up their patterns of sitting more frequently than if they were not self-monitoring. While this may be a positive outcome within "measurement as intervention" type studies (such as the use of self-monitoring for promoting behavior change), it is undesirable in contexts where the primary goal is to accurately capture information on habitual sedentary behavior.

\section{Objective Measures of Sedentary Behavior}

Technological advancements in recent years have led to increased accessibility to, and use of, objective measurement of sedentary behavior. Objective measures of sedentary behavior can be categorized into two types: energy expenditure devices and posture classification devices (Granat, 2012). Importantly, most of these devices use the same underlying technology (accelerometry) but use different algorithms to interpret the data to estimate either energy expenditure or body position. Energy expenditure devices, which generally comprise accelerometers such as the ActiGraph GT3X (ActiGraph, Pensacola, FL), are typically worn on the waist or wrist. They measure human movement and provide the magnitude of the acceleration within set time periods (epochs), or as a continuous data stream 
at frequencies such as $50 \mathrm{~Hz}$. This is then translated into proprietary activity counts (i.e., the underlying technology is the collection of streaming acceleration data and subsequent conversion into counts in time epochs using proprietary algorithms and software). The thresholds of activity counts that correspond to the energy expenditure levels associated with sedentary behavior ( $\leq 1.5 \mathrm{METs}$ ) vary somewhat, but a threshold of less than $100 \mathrm{cpm}$ has generally been used with vertical axis data from the ActiGraph (Matthews et al., 2008). As such devices are now capable of measuring acceleration in three axes, research is ongoing to develop equivalent cutpoints using the three-axis vector magnitude.

Posture classification devices, such as the activPAL (PAL Technologies Ltd, Glasgow, UK), provide data on absolute body positions or status of human movement. Similar to energy expenditure devices, the derived data (e.g., incline of the thigh) are continuous and based on body segment acceleration, and decision criteria are used to decide whether to classify incline of the instrument as horizontal (sitting/lying) or vertical (standing). The activPAL device is capable of monitoring shifts in posture (e.g., moving from sitting or lying to standing and vice versa) by assessing motion in the vertical, horizontal, and anterioposterior planes, functions as an inclinometer, and provides outputs on three types of postural activities (i.e., sitting/lying, standing, and stepping) using an event file (Grant, Ryan, Tigbe, \& Granat, 2006). Thus, time stamped data record when the wearer transitions from one mode (sit/lie, stand or step) to another. The exact angle of the thigh that corresponds to transitioning from a sitting to standing position, and that corresponds to transitioning from standing to sitting, are different, as applied by the proprietary algorithms. Specifically, a differential threshold is applied, where the angle determining a sit-to-stand transition is closer to vertical than the angle used to determine a stand-to-sit transition. Manufacturer software is then used to reduce data from the event file into summary data on time spent sitting/lying, standing, and 
walking, total steps, and other outcome variables that are facilitated by the time-stamped event recordings such as number of bouts in each mode, average length of each bout for each mode, number of transitions from sedentary to upright position, and time patterning (time of day associated with different behavior patterns).

Regardless of whether a primarily energy-expenditure-driven instrument or a primarily posture-driven instrument is used, similar processes underlie the generation of data from objective devices, as represented in Table 1. Error can be introduced at any stage of the process, from the measurement of raw acceleration in the initial stages, through the application of firmware and software algorithms for preliminary processing, to the decision rules applied in the latter stages. The latter includes such decisions as what constitutes a valid day of wear, what is the minimum required days to constitute reliable and valid data, and minimum bout length criteria. From a strict measurement perspective, errors at only the first stage of the process could be labelled as measurement error. However, because acceleration is not the primary construct of interest, we propose that any of the subsequent stages of the process should be considered as possible sources of error. As we show later in this paper, decisions such as what cutpoints to apply, definitions of minimal bout lengths, and parameters for defining sedentary breaks all can influence the variability in outcome variables representing the various dimensions of sedentary behavior represented by the SITT acronym. Similar to many other areas of kinesiology such as the prediction of maximal aerobic fitness or the prediction of body composition components, all sources of variability that are not attributable to true score variability should be considered, including aspects such as data reduction techniques, application of software algorithms and rules associated with valid wear time.

Objective measures of sedentary behaviors are increasingly used as they are believed 
to provide more valid and reliable estimates of total time spent in sedentary behaviors in a free-living environment compared to subjective measures of sedentary behaviors (Atkin et al., 2012; Healy, Clark, et al., 2011). They also provide more detailed data on temporal pattern. There are, however, several challenges of using objective measures.

First, both types of sedentary behavior monitoring devices (accelerometry-based motion sensors and posture sensors) have limited functional abilities for measuring sedentary behavior in accordance with the prevailing conceptual definition of sedentary behavior. This may lead to biased estimates of time spent in sedentary behaviors in a free-living environment. For instance, some accelerometers may mis-classify as sedentary behavior activities such as quiet standing or some light-intensity activities in a standing position where the activity counts for those activities are below 100 per minute (Granat, 2012; Marshall \& Merchant, 2013). Conversely, activPAL does not directly implement the definition regarding energy expenditure $(\leq 1.5 \mathrm{METs})$ in measuring sedentary behaviors and would classify some active sitting or lying activities with high energy expenditure ( $>1.5$ METs) such as weight lifting as sedentary behaviors.

Notably, much health-related research will combine the investigation of sedentary behaviors and physical activity behaviors. The conceptual representation of sedentary behaviors as part of a spectrum of physical behaviors including sleep, sedentary behavior and physical activity, as previously described by Tremblay et al. will probably become standard within future public health research. This theoretical perspective is taking hold via organizations such as the International Society for the Measurement of Physical Behaviour (see http://www.ismpb.org/) and disseminated bi-annually at the International Congress of Ambulatory Monitoring of Physical Activity and Movement. In terms of objective monitoring of the spectrum of physical behaviors, it is probably fair to say that the activPAL (which is 
unique in currently being the only such commercially-available device) is more accurate at measuring the sedentary end of the spectrum than the physical activity end of the spectrum (because currently, physical activity data from the activPAL is limited to step counts and stepping frequency, or cadence). Conversely, waist-worn accelerometers are more accurate at measuring the physical activity end of the spectrum than the sedentary end (because they do not accurately measure posture).

However, recent developments reveal that both instruments, in association with advances in associated software and algorithms, are improving the accuracy of measuring a variety of physical behaviors. For example, the ActiGraph currently incorporates an inclinometer for use when worn at the waist. Carr \& Mahar evaluated its accuracy for classifying inactive lying and sitting and inactive standing, and found classification accuracy to be only $61 \%-67 \%$. Interesting research has been conducted recently on the use of wristworn accelerometry for identifying sedentary behaviors. Rowlands et al. (2014) investigated the classification accuracy of the GENEActive triaxial accelerometer for determining posture, using the activPAL as the criterion measure. In three small but diverse samples, classification accuracy from the wrist-worn GENEActive for sitting/lying and standing was generally moderate to high, as evidenced by inter-instrument correlations, kappa, and mean differences. Some of the results were not as positive, but this method is still at the developmental stage and shows promise for measuring the broader spectrum of physical behaviors.

Second, the appropriateness of the activity counts threshold between sedentary behavior and light-intensity activity is of particular importance (Kim, Lee, Peters, Gaesser, \& Welk, 2014; Kozey-Keadle, Libertine, Lyden, Staudenmayer, \& Freedson, 2011). The activity count threshold of $100 \mathrm{cpm}$ for ActiGraph single-axis data is generally accepted in adults, but the validity evidence and justification of this threshold is quite limited (Atkin et al., 2012; 
Matthews et al., 2008). Kozey-Keadle et al. (2011) evaluated different thresholds of activity counts $(50,100,150,200$, and $250 \mathrm{cpm})$ in a sample of 20 overweight, inactive office workers. They found that compared to direct observation, the ActiGraph GT3X $100 \mathrm{cpm}$ threshold underestimated sitting time by approximately 5\% while the $150 \mathrm{cpm}$ threshold demonstrated the lowest percent bias (1.8\%). Kim et al. (2014) evaluated the criterionreferenced validity of several sedentary thresholds $(100,200,300,500,800$, and 1,100 cpm) using the ActiGraph GT3X placed on the hip and wrist for children while performing a series of prescribed activities ranging from sedentary to vigorous. Criterion classification was based on compendium MET values and each activity was dichotomized as either sedentary or nonsedentary. Results supported the use of a $<100 \mathrm{cpm}$ threshold with the highest classification accuracy at the hip (93\% agreement; Cohen's $\kappa=.81$; sensitivity $=94 \%$; specificity $=93 \%)$. Another unique finding of their study was that the thresholds were highly variable between hip and wrist, suggesting that location must be considered in applying an activity count threshold for sedentary behavior, as is the case for determining threshold for physical activity intensities. This is especially critical as large-sample surveys such as NHANES have switched to the wrist-worn position in order to increase compliance. This appears to have worked, as compliance increased from $40 \%-70 \%$ in the $2003-2004$ NHANES cycle to $70 \%$ $80 \%$ in the 2011-2012 cycle (Troiano, McClain, Brychta, \& Chen, 2015). NHANES is probably the most widely-used data set for epidemiological publications on physical activity and health, with over 3,000 publications containing the term NHANES in their title. As the switch to a wrist-worn position in NHANES has been so successful in improving adherence and wear time, it is reasonable to assume that the wrist-worn position will continue to be used (and may increasingly be used) in future research on physical activity and sedentary behaviors. 
Third, wear time can significantly influence estimation of sedentary behavior. This is likely to be greater for sedentary behavior than for physical activity, as sedentary behavior comprises more daily time than total physical activity time, and also than time spent in any physical activity intensity (light, moderate, vigorous, etc.). While it is commonly accepted that the minimum wear time for accelerometer derived physical activity is $\geq 10$ hours per day (h/day), there are no guidelines for minimum wear time for sedentary behavior. Herrmann, Barreira, Kang and Ainsworth (2013) used a semi-simulation approach in 124 adults participating in a workplace walking intervention to investigate the effect of wear time on various physical activity intensities and on sedentary time. They found that time spent in sedentary behavior $(<100 \mathrm{cpm})$ was $28 \%$ less $(406.5 \mathrm{~min}$ vs. $567.3 \mathrm{~min})$ when $10 \mathrm{~h}$ /day was compared with a reference of $14 \mathrm{~h} /$ day of accelerometer wear time. In comparison, although physical activity underestimation (combining light, moderate, and vigorous intensity physical activity) was similar proportionally (29\%), it constituted a smaller absolute discrepancy (76 min vs. $161 \mathrm{~min}$ for sedentary behavior). Moderate-to-vigorous physical activity underestimation was even smaller (14 min). This shows a substantially greater absolute underestimation of free-living sedentary behavior than for physical activity. Herrmann, Barreira, Kang and Ainsworth (2014) subsequently conducted a similar analysis on the 20052006 NHANES dataset and found a similar pattern to the previous study; allowing data with $10 \mathrm{~h}$ /day of wear time led to missing $30 \%$ of sedentary time $(<100 \mathrm{cpm})$ compared to the criterion $14 \mathrm{~h} /$ day of wear time.

Both studies support longer accelerometer wear time (i.e., greater than $13 \mathrm{~h} /$ day) to ensure accurate estimates of daily sedentary behavior. This is a considerable challenge for studies using waist-mounted accelerometers as compliance for longer wear time is poor. For example, Tucker determined that within the 2005-2006 NHANES data cycle, compliance 
with a minimum wear-time of 12 hours was $18 \%$ (7-day minimum), 50\% (5-day minimum), and $72 \%$ (3-day minimum) compared to compliance values for 10 hours of 33\% (7-day minimum), 67\% (5-day minimum), and 83\% (3-day minimum). Overall, this corresponded to data loss of $11 \%-17 \%$ for a $12-\mathrm{hr}$ minimum wear time compared to a $10-\mathrm{hr}$ minimum wear time.

One solution may be to individually impute data from shorter wear times to a defined "maximum day". For example, if the maximum day were defined as 16 hours of wake time, and sedentary time from 10 hours of wear was determined to be 8.5 hours (i.e., $85 \%$ of wear time), imputed sedentary time would be 13.6 hours ( $85 \%$ of 16 hours). Such calculations are based on an assumption of "equal qualities" in the missing data - in other words, an assumption that sedentary behavior during non-wear time was similar to that during wear time. There is some evidence to support this assumption. At the sample level, in the Herrmann et al. (2014) study, percent time spent being sedentary was 54.9\% for a $14-\mathrm{hr}$ minimum wear time, compared to $54.5 \%$ for $13 \mathrm{hr}, 54.3 \%$ for $12 \mathrm{hr}, 54.2 \%$ for $11 \mathrm{hr}$, and $54.0 \%$ for $10 \mathrm{hr}$. This does not necessarily translate to the level of the individual participant, and additional semi-simulation research is needed to assess the validity of such imputation.

Recent Developments in Sedentary Behavior Measurement-Related Research

Despite increased awareness of the importance of sedentary behavior, little attention has been paid to validity of the bout duration of sedentary behavior measurement. Although sedentary behavior is defined as prolonged sitting or reclining (Owen et al., 2009), the majority of studies that examined objectively measured sedentary behavior using accelerometers have used a 1-minute bout as the minimum length of duration for valid sedentary minutes (Clark et al., 2011; Healy, Matthews, Dunstan, Winkler, \& Owen, 2011; Maher, Mire, Harrington, Staiano, \& Katzmarzyk, 2013). This may be a legacy effect, as 
most such studies have used NHANES data, which was based on early ActiGraph accelerometers that were initialized to collect data in 1-min epochs. Aggregating data from 1min epochs to estimate total time spent in sedentary behavior from accelerometer data may lead to inclusion of significant time spent in 'sporadic sedentary behavior' that is not necessarily 'prolonged sedentary behavior'. It can mask the "interruptions" component of sedentary behavior - a single 30-minute bout of sitting is treated the same as 15 separate 2minute bouts of sitting. The operationalization of sedentary time from 1-min epoch data may therefore not be congruent with what has been defined at the conceptual level (i.e., from a health perspective, prolonged periods of continuous sitting are more harmful to health). This is likely an issue for all types of objective measures of sedentary behavior (motion sensors and posture sensors) and should be considered very carefully when deciding on data reduction procedures.

In a study to examine patterns of sedentary behavior among US adults in 2003-2006 NHANES, Kim, Welk, Braun, and Kang (2015) found that the current algorithm to identify sedentary behavior (a minimum of 1-min bout for sedentary time) in accelerometry data may not be appropriate to obtain valid measurements of sedentary behavior that are compliant with the current definition of sedentary behavior. The study examined the influences of different bout periods (i.e., 1, 2-4, 5-9, 10-14, 15-19, 20-24, 25-29, and 30-min) of sedentary behaviors on health outcomes, and the results showed that sedentary minutes obtained by a minimum of 5-min bouts yielded a better model-data fit to predict cardiovascular risk factors.

Studies have shown that sedentary breaks are positively associated with health outcomes, independent of total sedentary minutes (Healy, Dunstan, Salmon, Shaw, et al., 2008; Healy, Matthews, et al., 2011). Healy, Dunstan, Salmon, Cerin, et al. (2008) presented 
the idea of two individuals with similar total sedentary time. The "prolonger" accumulated his time in long, continuous bouts with few interruptions, while the "breaker" accumulated her time via multiple, shorter bouts with frequent interruptions. The findings of Healy, Dunstan, Salmon, Shaw, et al. (2008) and Healy, Matthews, et al. (2011) have prompted many sedentary behavior researchers to modify patterns of sedentary time accumulation by breaking up prolonged sedentary time as an explicit intervention strategy (Owen et al., 2010). It is still, however, unclear what a "valid" sedentary break is. In this case, validity does not refer to accuracy of measurement but appropriateness of the measurement - in order to determine what is a "valid" break, we need to determine what break characteristics are related to health benefits. A break in sedentary time is typically operationalized by counting every transition point from a sedentary to active phase (e.g., from $<100 \mathrm{cpm}$ to $\geq 100 \mathrm{cpm}$; Healy, Dunstan, Salmon, Shaw, et al., 2008). When we measure breaks in sedentary time in a freeliving environment using common data processing criteria, we do not differentiate whether such a postural transition is a true break in sedentary time or simply the end of a brief bout of sedentary behavior. For example, a brief rest of 1-2 min during a period of activity currently is classified as a break in sedentary behavior, whereas it is truly more a brief break in an activity bout!

Kim et al. (2015) investigated the construct validity of current methods of sedentary break measurement. In their study, they regressed various permutations of sedentary break computation on four health indicators (waist circumference, HDL-C, fasting triglyceride, and body mass index). Primarily, the purpose was to test the effect of differentiating between breaks that follow short bouts of sedentary behavior (operationally defined as $<5$ min and $<$ $10 \mathrm{~min}$ ), and breaks that follow longer bouts of behavior (operationally defined as $\geq 5 \mathrm{~min}$ and $\geq 10 \mathrm{~min}$ ). The authors found that the number of sedentary breaks was positively 
(beneficially) associated with health outcomes when delimited to breaks that followed a brief bout of sedentary behavior, but negatively (detrimentally) associated with health outcomes when limited to breaks that followed a longer sedentary bout duration. This clearly indicates that when investigating sedentary breaks, we should not apply the prevailing "one-size-fits-all" approach in which any postural transition following a period of sedentary behavior is counted as a break, regardless of how short the sedentary bout was (typically, anything of at least 1 min is currently counted as valid). From the study, it appears that different methods for computing a sedentary break result in variables that have different implications for health. The apparent finding that "more frequent" breaks in prolonged sitting are detrimentally associated with health seems counterintuitive. However, it simply reflects the fact that the number of breaks following prolonged sedentary bouts serves as a proxy for prolonged sitting (i.e., a break can only occur if a prolonged bout occurs). Interestingly, the total number of sedentary breaks was identical or slightly less than the accrued number of sedentary bouts of at least 1-min bout duration. In other words, counting the number of transition points from a sedentary to active phase would produce a very similar number compared to the number of sedentary bouts (Kim et al., 2015). This finding indicates that we might be incorrectly measuring the conceptual definition of valid sedentary breaks (interruptions in sedentary time) using the current method of processing data to arrive at sedentary breaks. Further research is needed to improve the validity of objectively measured sedentary breaks.

Besides temporal issues related to sedentary bout length and sedentary breaks, there are broader temporal conundrums in the assessment of total daily sedentary time. Unlike MVPA, which is largely temporally independent of waking time, sedentary time is much more temporally dependent on wake time. The pie chart in Figure 1 illustrates why. Physical behavior during total daily time ( $24 \mathrm{hr}$ ) can be broken up into four types of behaviors. A small 
time period is generally spent in MVPA. For example, from national accelerometry data in the US, adults in various age and sex categories spend on average only 5.4 to $42.8 \mathrm{~min} /$ day in MVPA (Troiano et al., 2008), even using very liberal criteria (not applying a restrictive 10min bout condition). This corresponds to only $0.4 \%$ to $3.0 \%$ of each daily 24 -hr cycle. Conversely, mean sleep time in a similar sample of adults ranged from 6.7 to $7.4 \mathrm{hr}$, or $28 \%$ to $31 \%$ of a daily $24-\mathrm{hr}$ cycle (Ram, Seirawan, Kumar, \& Clark, 2010). Also using NHANES accelerometry data, Matthews et al. (2008) determined average time spent by adults in sedentary behavior $(\mathrm{cpm}<100)$ to be $7.5 \mathrm{hr}$ to $9.3 \mathrm{hr}$, or $31 \%$ to $39 \%$ of a daily $24-\mathrm{hr}$ cycle, adjusted for wear time. These proportions are approximated in Figure 1, from which it is evident that MVPA comprises a trivial proportion of the 24-hr day, and sleep, light intensity physical activity and sedentary time comprise approximately one-third each of total daily time.

Certain mathematical conclusions can be derived from this information. First, changes in MVPA (and errors in measurement of MVPA) will have no meaningful displacement effect on time spent in sleep, light intensity physical activity and sedentary behavior. Second, if sleep is held constant, displacement of sedentary time will be directly related to changes in light intensity physical activity time. This latter conundrum has been addressed recently from both a measurement perspective and a behavior modification perspective (i.e., in order to reduce or break up prolonged sedentary time, behavioral interventions often target increasing light intensity physical activity). From a measurement or statistical perspective, the effect of reduction in sedentary time on health, and relationships between sedentary time and health, are collinearly associated with similar effects (or relationships) between light intensity physical activity and health. This was demonstrated by Maher, Olds, Mire, and Katzmarzyk (2014), who found that when adjusted for total physical activity (i.e., the sum of light, 
moderate and vigorous intensity physical activity), most correlations between sedentary behavior and several cardiometabolic markers effectively disappeared. Third, because sleep (a physical behavior that is positively related to health) varies among individuals and is directly mathematically related to sedentary time, estimates of sedentary time should be adjusted to take sleep into account. Pedisic (2014) reviewed 54 studies investigating relationships between sedentary behavior and health, of which only two studies, investigating TV time and health, adjusted for sleep time. In his thorough review of the underlying statistical issues, he proposed the Activity Balance Model, a new theoretical framework for combining the investigation of sedentary behavior and health with sleep duration, standing time, light intensity physical activity and MVPA. While there is currently little empirical evidence to support this framework, it seems inevitable that the measurement and investigation of sedentary behavior will be inextricably linked with the broader spectrum of physical behaviors.

Through rapid technological advancements and collaborations across disciplines, new analytic and modeling approaches to classifying different types of behaviors have been developed and tested. Lyden, Kozey-Keadle, Staudenmayer, and Freedson (2014) introduced the sojourn method, a machine learning technique that combines artificial neural networks with decision tree analysis, and found that two sojourn methods (sojourn-1 Axis and sojourn3 Axis) improved the estimation of sedentary time from a single, hip-mounted accelerometer compared to direct observation over 3 days of free living in 13 adults. More validation studies regarding the application of analytic and modeling approaches in free-living environments are needed.

\section{Conclusion}


In this paper we have attempted to set the scene for subsequent evidence-based papers evaluating methods for assessing sedentary behavior. In population-based observational studies to monitor sedentary behavior, subjective measures have been preferred for their efficiency and practicality; however, several disadvantages of subjective measures impede the reliable and valid estimation of sedentary behavior in free-living environments. Furthermore, while objective measures of physical activity have been shown to provide more reliable and valid estimates for sedentary behavior compared to subjective measures, they are still limited in their ability to obtain accurate measurements that comply with the current definition of sedentary behavior. Specifically, no current widely-available objective tool accurately detects both posture and energy expenditure, and none accurately measures the full spectrum of physical behaviors (from sleep to vigorous intensity physical activity).

In this paper, different methods have been described for assessing sedentary behavior, but no specific method can be identified as the best method for all purposes. Each method has its own advantages and disadvantages, which must be carefully considered before selecting a measure. New analytic and modeling approaches for translating raw accelerometer data to classify different types of sedentary behavior show considerable promise; yet challenges regarding the application of analytic and modeling approaches in free-living environments still need to be addressed. Regardless of the sedentary behavior measure chosen, researchers must be aware of all possible sources of error inherent to each technique and attempt to minimize those errors thereby increasing the validity of the outcome data.

We present below specific recommendations based on the current evidence and thinking presented in this paper:

- Subjective measures are primarily suitable for providing general summary information. They are most suited to use in large-sample descriptive studies, 
with the caveat that they provide less reliable estimates of sedentary behavior than objective measures. They can be used to provide useful information on context and type of behavior and therefore are a valuable adjunct method for use with objective measures, in order to understand behavioral aspects and thereby inform interventions.

- Until technological advances facilitate the combination of both methods in a single instrument, posture-focused instruments should be used where sedentary behavior is the primary outcome of interest and energy-expenditurefocused instruments should be used where differentiating between intensities of physical activity is the primary goal.

- Wear time influences estimates of sedentary behavior more than for estimating other physical behaviors. To resolve this problem, either stricter wear time criteria must be applied or missing data should be imputed to at least reflect a "standard day". Further semi-simulation studies are needed in order to evaluate the effect of various imputation methods on accuracy of full-day estimates of sedentary behavior.

- Because of the mathematical interdependence of sedentary time and sleep time, and the fact that sleep time is generally beneficial to health, estimates of sedentary time should preferably be adjusted to reflect accurately-determined wake time, possibly assessed via a wake and sleep log.

- When aggregating sedentary time, a minimum bout length of at least 5 continuous minutes should be used from waist-worn accelerometer devices. 
Issues and Challenges 24

- Current methods of computing sedentary breaks incorrectly measure the conceptual definition of valid sedentary breaks. Thus, caution is needed when interpreting results about sedentary breaks in relation to health outcomes, and future research is warranted to improve the validity of objectively measured sedentary breaks. Parameters relating to the length of the sedentary bout preceding a break and the length of the break itself also need to be considered.

- Machine-learning techniques show promise for estimating sedentary time from waist-mounted accelerometry, but as with similar methods for analyzing physical activity data, we seem to be a long way from widespread incorporation of these methods into software for processing free-living acceleration data. 


\section{References}

American College of Sports Medicine. (1978). ACSM Position statement on the recommended quantity and quality of exercise for developing and maintaining fitness in healthy adults. Medicine and Science in Sports and Exercise, 10(3), vi-x.

Atkin, A. J., Gorely, T., Clemes, S. A., Yates, T., Edwardson, C., Brage, S., . . Biddle, S. J. H. (2012). Methods of measurement in epidemiology: Sedentary behavior. International Jounal of Epidemiology, 41, 1460-1471.

Australian Government Department of Health. (2014). Australia's physical activity and sedentary behaviour guidelines: Adults. Canberra, Australia: Author.

Brown, W. J., Bauman, A. E., Bull, F. C., \& Burton, N. W. (2012). Development of evidencebased physical activity recommendations for adults (18-64 years): Final report. Canberra, Australia: Australian Government Department of Health.

Canadian Society for Exercise Physiology. (2012). Canadian physical activity guidelines; Canadian sedentary behaviour guidelines. Ottawa, Canada: Author.

Campbell, D. T., \& Stanley, J. C. (1966). Experimental and quasi-experimental designs for research. Chicago: Rand McNally.

Carr, L. J., \& Mahar, M. T. (2012). Accuracy of intensity and inclinometer output of three activity monitors for identification of sedentary behavior and light-intensity activity. Journal of Obesity, 2012, Article ID 460271.

Centers for Disease Control and Prevention. (1993). Prevalence of sedentary lifestyle Behavioral Risk Factor Surveillance System, United States, 1991. Morbidity and Mortality Weekly Report, 42(29), 576-579.

Chastin, S. F. M., Schwarz, U., \& Skelton, D. A. (2013). Development of a consensus taxonomy of sedentary behaviors (SIT): Report of Delphi round 1. PloS ONE, 8(12), 
e82313. doi: 10.1371/journal.pone.0082313

Chau, J. Y., van der Ploeg, H. P., Dunn, S., Kurko, J., \& Bauman, A. E. (2012). Validity of the Occupational Sitting and Physical Activity Questionnaire. Medicine and Science in Sports and Exercise, 44, 118-125.

Clark, B. K., Healy, G. N., Winkler, E. A., Gardiner, P. A., Sugiyama, T., Dunstan, D. W., .. . Owen, N. (2011). Relationship of television time with accelerometer-derived sedentary time: NHANES. Medicine and Science in Sports and Exercise, 43, 822-828.

Clemes, S. A., David, B. M., Zhao, Y., Han, X., \& Brown, W. (2012). Validity of two selfreport measures of sitting time. Journal of Physical Activity and Health, 9, 533-539.

Dunton, G. F., Liao, Y., Intille, S. S., Spruijt-Metz, D., \& Pentz, M. (2011). Investigating children's physical activity and sedentary behavior using ecological momentary assessment with mobile phones. Obesity, 19, 1205-1212.

Granat, M. H. (2012). Event-based analysis of free-living behavior. Physiological Measurement, 33, 1785-1800.

Grant, P. M., Ryan, C. G., Tigbe, W. W., \& Granat, M. H. (2006). The validation of a novel activity monitor in the measurement of posture and motion during everyday activities. British Journal of Sports Medicine, 40, 992-997.

Healy, G. N., Clark, B. K., Winkler, E. A. H., Gardiner, P. A., Brown, W. J., \& Matthews, C. E. (2011). Measurement of adults' sedentary time in population-based studies. American Journal of Preventive Medicine, 41, 216-227.

Healy, G. N., Dunstan, D. W., Salmon, J., Cerin, E., Shaw, J. E., Zimmet, P. Z. , . . Owen, N. (2008). Breaks in sedentary time: Beneficial associations with metabolic risk. Diabetes Care, 31, 661-666. doi:10.2337/dc07-2046

Healy, G. N., Dunstan, D. W., Salmon, J., Shaw, J. E., Zimmet, P. Z., \& Owen, N. (2008). 
Television time and continuous metabolic risk in physically active adults. Medicine and Science in Sports and Exercise, 40, 639-645.

Healy, G. N., Matthews, C. E., Dunstan, D. W., Winkler, E. A., \& Owen, N. (2011). Sedentary time and cardio-metabolic biomarkers in US adults: NHANES 2003-06. European Heart Journal, 32, 590-597.

Herrmann, S. D., Barreira, T. V., Kang, M., \& Ainsworth, B. E. (2013). How many hours are enough? Optimal accelerometer wear time to reflect daily activity. Journal of Physical Activity and Health, 10, 742-749.

Herrmann, S. D., Barreira, T. V., Kang, M., \& Ainsworth, B. E. (2014). Impact of accelerometer wear time on physical activity data: A NHANES semisimulation data approach. British Journal of Sports Medicine, 48, 278-282.

Kerr, J., Marshall, S. J., Godbole, S., Chen, J., Legge, A., Doherty, A. R. , . . Foster, C. (2013). Using the SenseCam to improve classifications of sedentary behavior in freeliving settings. American Journal of Preventive Medicine, 44, 290-296.

Kim, Y., Barry, V. W., \& Kang, M. (2015). Validation of objective monitoring devices for the assessment of sedentary behavior: Implication of minimum sedentary bout durations. Measurement in Physical Education and Exercise Science, 19, xxx-xxx.

Kim, Y., Lee, J., Peters, B. P., Gaesser, G. A., \& Welk, G. J. (2014). Examination of different accelerometer cut-points for assessing sedentary behaviors in children. PLoS ONE, 9(4), e90630. doi:10.1371/journal.pone.0090630

Kim, Y., Welk, G. J., Braun, S. I., \& Kang, M. (2015). Extracting objective estimates of sedentary behavior from accelerometer data: Measurement considerations for surveillance and research applications. PLoS ONE, 10(2), e0118078. doi:10.1371/journal.pone.0118078 
Kozey-Keadle, S., Libertine, A., Lyden, K., Staudenmayer, J., \& Freedson, P. S. (2011). Validation of wearable monitors for assessing sedentary behavior. Medicine and Science in Sports and Exercise, 43, 1561-1567.

Lyden, K., Kozey-Keadle, S., Staudenmayer, J., \& Freedson, P. S. (2014). A method to estimate free-living active and sedentary behavior from an accelerometer. Medicine and Science in Sports and Exercise, 46, 386-397.

Maher, C. A., Mire, E., Harrington, D. M., Staiano, A. E., \& Katzmarzyk, P. T. (2013). The independent and combined associations of physical activity and sedentary behavior with obesity in adults: NHANES 2003-06. Obesity, 21, E730-737. doi: 10.1002/oby.20430

Maher, C. A., Olds, T., Mire, E., \& Katzmarzyk, P. T. (2014). Reconsidering the sedentary behavior paradigm. PloS ONE, 9(1), e86403. doi: 10.1371/journal.pone.0086403

Marshall, A. L., Miller, Y. D., Burton, N. W., \& Brown, W. J. (2010). Measuring total and domain-specific sitting: A study of reliability and validity. Medicine and Science in Sports and Exercise, 42, 1094-1102.

Marshall, S. J., \& Merchant, G. (2013). Advancing the science of sedentary behavior measurement. American Journal of Preventive Medicine, 44, 190-191.

Matthews, C. E., Chen, K. Y., Freedson, P. S., Buchowski, M. S., Beech, B. M., Pate, R. R. , . . Troiano, R. P. (2008). Amount of time spent in sedentary behaviors in the United States, 2003-2004. American Journal of Epidemiology, 167, 875-881.

Owen, N., Bauman, A., \& Brown, W. (2009). Too much sitting: A novel and important predictor of chronic disease risk? British Journal of Sports Medicine, 43, 81-83.

Owen, N., Healy, G, N., Matthews, C. E., \& Dunstan, D. W. (2010). Too much sitting: The population-health science of sedentary behavior. Exercise and Sport Sciences Reviews, $38,105-113$. 
Paffenbarger, R. S., Hyde, R., Wing, A. L., \& Hsieh, C-C. (1986). Physical activity, all-cause mortality, and longevity of college alumni. New England Journal of Medicine, 314, 605613.

Pate, R. R., O'Neill, J. R., \& Lobelo, F. (2008). The evolving definition of "Sedentary". Exercise and Sport Sciences Reviews, 36, 173-178.

Pate, R. R., Pratt, M., Blair, S. N., Haskell, W. L., Macera, C. A., Bouchard, C. , . . Wilmore, J. H. (1995). Physical activity and public health: A recommendation from the Centers for Disease Control and Prevention and the American College of Sports Medicine. JAMA, 273, 402-407.

Pedisic, Z. (2014). Measurement issues and poor adjustments for physical activity and sleep undermine sedentary behaviour research - the focus should shift to the balance between sleep, sedentary behaviour, standing and activity. Kinesiology, 46, 135-146.

Ram, S., Seirawan, H., Kumar, S. K. S., \& Clark, G. T. (2010). Prevalence and impact of sleep disorders and sleep habits in the United States. Sleep and Breathing, 14, 63-70

Rosenberg, D. E., Norman, G. J., Wagner, N., Patrick, K., Calfas, K. J., \& Sallis, J. F. (2010). Reliability and validity of the Sedentary Behavior Questionnaire (SBQ) for adults. Journal of Physical Activity and Health, 7, 697-705.

Rosenberger, M. (2012). Sedentary behavior: Target for change, challenge to assess. International Journal of Obesity, Suppl2, S26-S29. doi: 10.1038/ijosup.2012.7

Rowlands, A. V., Olds, T. S., Hillsdon, M., Pulsford, R., Hurst, T. L., Eston, R. G. , . . Langford, J. (2014). Assessing sedentary behavior with the GENEActiv: Introducing the sedentary sphere. Medicine and Science in Sports and Exercise, 46, 1235-1247.

Sallis, J. F., \& Saelens, B. E. (2000). Assessment of physical activity by self-report: Status, limitations, and future directions. Research Quarterly for Exercise and Sports, 72(2S), 


\section{S-14S.}

Salmon, J., Arundell, L., Hume, C., Brown, H., Hesketh, K., Dunstan, D. W. , . . Crawford, D. (2011). A cluster-randomized controlled trial to reduce sedentary behavior and promote physical activity and health of 8-9 year olds: The Transform-Us! Study. BMC Public Health, 11, 759. doi:10.1186/1471-2458-11-759

Sedentary Behaviour Research Network. (2012). Letter to the Editor: Standardized use of the terms "sedentary" and "sedentary behaviours". Applied Physiology, Nutrition, and Metabolism, 37, 540-542.

Tremblay, M. S., Colley, R. C., Saunders, T. J., Healy, G. N., \& Owen, N. (2010). Physiological and health implications of a sedentary lifestyle. Applied Physiology, Nutrition, and Metabolism, 35, 725-740.

Tremblay, M. S., Warburton, D. E. R., Janssen, I., Paterson, D. H., Latimer, A. E., Rhodes, R. E. , ... Duggan, M. (2011). New Canadian physical activity guidelines. Applied Physiology, Nutrition and Metabolism, 36, 36-46

Troiano, R. P., Berrigan, D., Dodd, K. W., Mâsse, L. C., Tilert, T., \& McDowell, M. (2008). Physical activity in the United States measured by accelerometer. Medicine and Science in Sports and Exercise, 40, 181-188.

Troiano, R. P., McClain, J. J., Brychta, R. J., \& Chen, K. Y. (2015). Evolution of accelerometer methods for physical activity research. British Journal of Sports Medicine, 48, 1019-1023.

Tucker, J. (2010). Physical activity levels and cardiovascular disease risk among U.S. adults: Comparison between self-reported and objectively measured physical activity. Graduate Theses and Dissertations, Paper 11746.

US Physical Activity Guidelines Committee. (2008). Physical Activity Guidelines Advisory 
Committee report, 2008. Washington: DHSS. 
Table 1. Sources of error at three stages of the data collection and interpretation process.

\begin{tabular}{|c|c|c|c|}
\hline & $\begin{array}{c}\text { Primary stage: } \\
\text { Collection of raw data }\end{array}$ & $\begin{array}{c}\text { Secondary stages: } \\
\text { Conversion to interpretable scores }\end{array}$ & $\begin{array}{c}\text { Final stages: } \\
\text { Production of aggregate data }\end{array}$ \\
\hline Processes & $\begin{array}{l}\text { Hardware and firmware collect and } \\
\text { process high-frequency acceleration } \\
\text { signal }\end{array}$ & $\begin{array}{l}\text { Proprietary or custom software } \\
\text { applies algorithms to convert raw } \\
\text { signal to meaningful data }\end{array}$ & $\begin{array}{l}\text { Proprietary or custom software } \\
\text { applies decision rules to perform data } \\
\text { reduction tasks }\end{array}$ \\
\hline Outcomes & $\begin{array}{l}\text { Movement in } 1 \text { to } 3 \text { axes, usually } \\
\text { expressed in } g\end{array}$ & $\begin{array}{l}\text { Activity counts, energy } \\
\text { expenditure, body position }\end{array}$ & $\begin{array}{l}\text { Total volume (time) } \\
\text { Number of bouts } \\
\text { Length of bouts } \\
\text { Number of breaks }\end{array}$ \\
\hline
\end{tabular}




\section{Time spent in different physical behaviors}

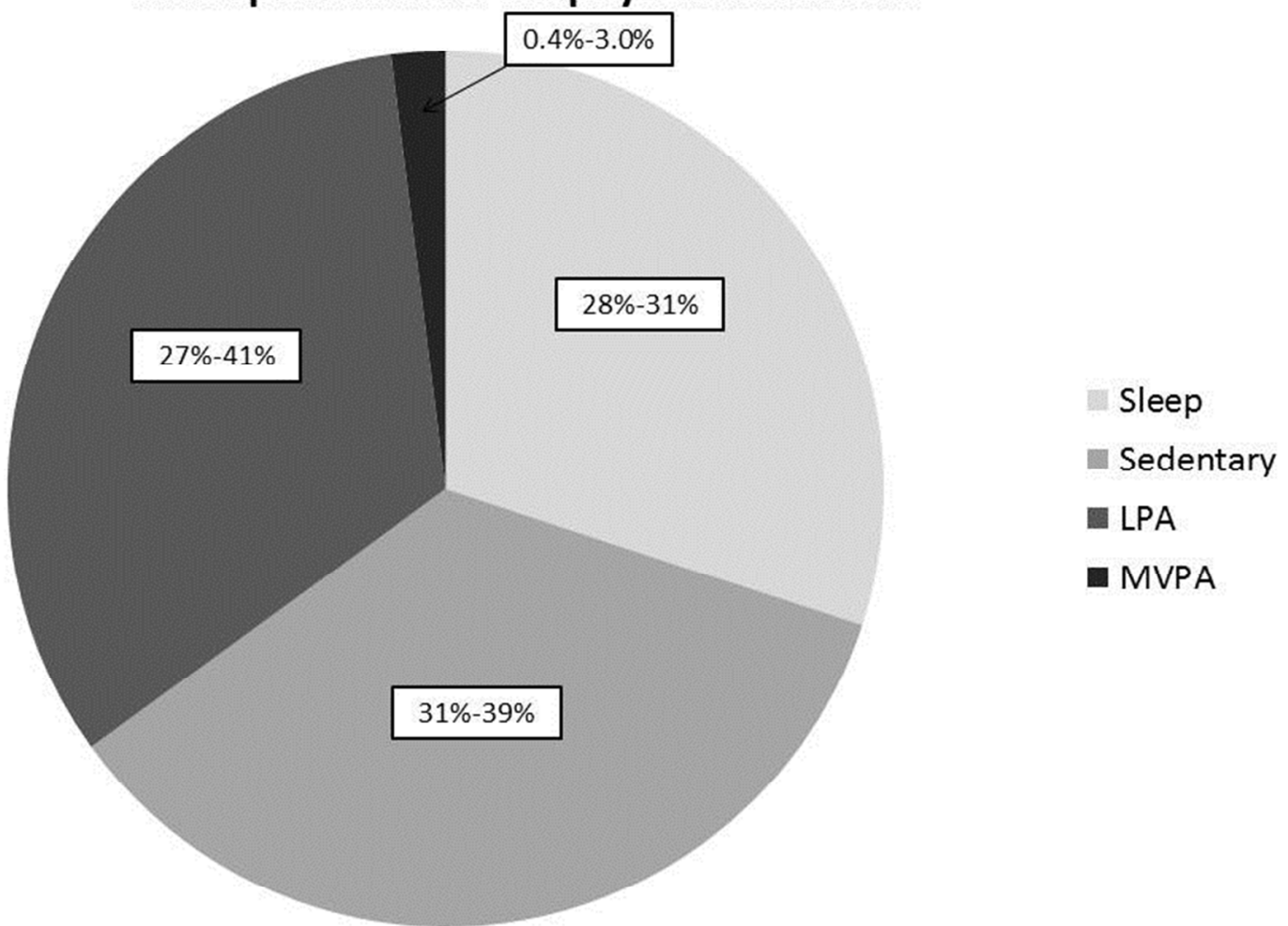

Note. Data are aggregated from the following sources: Matthews et al., 2008; Ram, Seirawan, Kumar \& Clark, 2010; Troiano et al., 2008

Figure 1. Time spent in different physical behaviors. 
Please proofread your manuscript carefully. For example:

\section{p. 20}

Use a comma to separate the authors:

“. . . 24-hr cycle (Ram, Seirawan, Kumar[,] \& Clark, 2010).

[Changed]

Avoid using the same term back-to-back:

"These proportions are approximated in Figure 1. From Figure 1, it is evident ..."

You may them into one sentence: "These proportions are approximated in Figure 1, which is evident ..."

[Modified. It now reads "... approximated in Figure 1, from which it is evident that..." - we feel this alleviates the concern about the term "Figure 1" being directly back-to-back, and we added the words "from ... it", in order to make grammatical sense).]

Use the "author, year" format:

"This was demonstrated by Maher, Olds, Mire, and Katzmarzyk (2014), who found that ..." [Changed]

Proofread the entire manuscript to make sure when to use "i.e.," or "e.g.," "i.e.," means "that is". For example:

It is $\$ 10$ if you order the ticket online; however, you need to pay double at the door (i.e., \$20). [Checked the entire manuscript as suggested.]

This latter conundrum has been addressed recently from both a measurement perspective and a behavior modification perspective ([e.g.g that to reduce sedentary time, one should target increasing light intensity physical activity).

[This has not been changed to e.g. The author of this section (DR) fully intended to use i.e., ("That is") the detail provided explains "the" exact situation to which I was referring (not an example), specifically it expands/explains the statement that behavioral interventions designed to disrupt prolonged sitting time tend to do so by introducing light physical activity such as getting up and walking to the water cooler. I have added some words to the sentence to remove any ambiguity about what I was trying to say here. The section now reads "This latter conundrum has been addressed recently from both a measurement perspective and a behavior modification perspective (i.e., in order to reduce or break up prolonged sedentary time, behavioral interventions often target increasing light intensity physical activity).]

“. . . when adjusted for total physical activity (i.e., light, moderate and vigorous intensity????)"

[Reworded to make this clearer: "when adjusted for total physical activity (i.e., the sum of light, moderate and vigorous intensity physical activity), ..."] 


\section{$\underline{\text { References }}$}

Make sure all the references are in APA format (e.g., use upper case after a colon, use sentence case for a title, use a comma to separate the journal, volume, and pages, use no period at the end of the "doi:xxxxx" and so on. Here are just a few examples:

Campbell, D. T., \& Stanley, J. C. (1966). Experimental and quasi-experimental designs for research. Chicago, IL: Rand McNally.

[This has not been changed. Chicago is one of a list of cities that APA says should not be followed by the state because they are well-known - we refer to APA $5^{\text {th }}$ edition, p. 217. However, we have italicized the book title, according to APA guidelines.]

Chastin, S. F. M., Schwarz, U., \& Skelton, D. A. (2013). Development of a consensus taxonomy of sedentary behaviors (SIT): Report of delphi round 1. PloS ONE[,] 8(12)[,] e82313. doi:10.1371/journal.pone.0082313

[Changed; also, Delphi has been capitalized in the manuscript, as this is a proper noun (named after the city of Delphi) and proper nouns should be capitalized in book titles and article titles.]

Healy, G. N., Dunstan, D. W., Salmon, J., Cerin, E., Shaw, J. E., Zimmet, P. Z. , . . Owen, N. (2008). Breaks in sedentary time: Beneficial associations with metabolic risk. Diabetes Care, 31(4)[,] 661-666. doi:10.2337/dc07-2046 [PMID:18252901???]

[Changed]

Kim, Y., Lee, J., Peters, B. P., Gaesser, G. A., \& Welk, G. J. (2014). Examination of different accelerometer cut-points for assessing sedentary behaviors in children. PLoS ONE[,] 9(4)[,] e90630. doi:10.1371/journal.pone.0090630

[Changed]

Kim, Y., Welk, G. J., Braun, S. I., \& Kang, M. (2015). Extracting objective estimates of sedentary behavior from accelerometer data: Measurement considerations for surveillance and research applications. PLoS ONE[,] 10(2)[,] e0118078. doi:10.1371/journal.pone.0118078

[Changed]

Maher, C. A., Mire, E., Harrington, D. M., Staiano, A. E., \& Katzmarzyk, P. T. (2013). The independent and combined associations of physical activity and sedentary behavior with obesity in adults: NHANES 2003-06. Obesity, 21[,] E730-737. doi:10.1002/oby.20430 [Changed]

Maher, C. A., Olds, T., Mire, E., \& Katzmarzyk, P. T. (2014). Reconsidering the sedentary behavior paradigm. PloS ONE[,] 9(1)[,] e86403. doi:10.1371/journal.pone.0086403 [Changed] 
US Physical Activity Guidelines Committee[.] (2008). Physical activity guidelines advisory committee report, 2008. Washington: DHSS. Retrieved from??????????

[This has not been changed. The citation information is exactly as suggested by the publisher of this document (i.e., no Web address). The document also is not a Web page (i.e., not changeable, and so retrieval date is not relevant. Also, The Physical Activity Guidelines Advisory Committee is a proper noun (title) and therefore should be capitalized even when formatted in sentence case.] 